\title{
DEVELOPING THE ACADEMIC THORACIC SURGEON: TEACHING SURGERY
}

William A. Baumgartner, MD

Peter S. Greene, MD
Teaching surgery can be a very gratifying experience for those of us involved in academic thoracic surgery. Fundamentals of a good residency program require that patients should always be placed in the highest priority. However, the residency program should also be committed to teaching as a priority. Creating the proper operating room environment is essential for optimal conduct of the operation. This environment is similar to that of the airline industry, which is known as crew or cockpit resource management. The design of a teaching program needs to have evaluation as one of its key elements. In addition to resident evaluation, it is also important to have faculty evaluation by the residents. The goal of any residency program should be to foster the development of the future leaders in our specialty. The information contained within this article represents the art and science of teaching thoracic surgery as applied by the faculty in the Division of Cardiac Surgery at The Johns Hopkins Hospital. (J Thorac Cardiovasc Surg 2000;119:S22-5)
$F_{8}^{\circ}$ or the surgeons and faculty in residency training programs, teaching residents is a daily function. Assisting residents in the operating room and watching them mature as both surgeons and technicians is 1 of the most gratifying roles we play in academic thoracic surgery. Although we participate in the training and education on a daily basis, we do not often think formally about how we teach surgery. The information contained within this article represents the art and science of teaching thoracic surgery as applied by the faculty in the Division of Cardiac Surgery at The Johns Hopkins Hospital.

\section{Fundamentals of a good residency program}

The fundamentals of a good residency program appear in Table I. The patient should always be placed in the highest priority. Placing the patient first must be the primary responsibility of both the attending surgeon and the resident. It is equally important to establish teaching and education as a priority within each residency program. Respect for the individual is a necessary component for any relationship to evolve. Similarly, it is the leader in the operating room who creates the proper and collegial environment. Without these 3 fundamentals, I do not think much teaching or

From The Johns Hopkins Hospital, Baltimore, Md.

Address for reprints: William A. Baumgartner, MD, Professor of Surgery, Cardiac Surgeon-in-Charge, The Johns Hopkins Hospital, 600 N Wolfe St, Blalock 618, Baltimore, MD 212874618.

Copyright $\odot 2000$ by The American Association for Thoracic Surgery. $0022-5223 / 2000 \$ 12.00+0 \quad \mathbf{1 2 / 0 / 1 0 4 7 2 1}$

doi:10.1067/mtc. 2000.104721 learning can be achieved. An adequate volume and variety of operative and diagnostic cases are necessary to provide the resident with a wide exposure of thoracic surgery. Evaluation and frequent feedback, both negative and positive, complete the fundamentals necessary for the development and maintenance of a good residency program.

Thoracic surgeons do a reasonably good job of teaching in the operating room. Fig 1 shows the responses of residents from various programs to a survey conducted by Dr Richard Shemin at a recent corporate-sponsored symposium. Of a maximum score of 4 , operating room teaching had a mean score of 3.5. This compared favorably with teaching performed at the bedside and in the clinic and didactic teaching. Although this isolated survey would suggest that thoracic residency programs are providing excellent operating room teaching, this still represents a mean score. There appears to be continued room for improvement.

\section{Teaching as a priority}

After their residency, a number of surgeons develop a love to operate independently or to "do their own cases." These surgeons should not be responsible for teaching surgery to residents. If, however, you want to be involved in a residency program, you have to commit to training residents. It seems to me that it has to be 1 of your core values.

Table II lists the core values of The Johns Hopkins University School of Medicine, which were recently reexamined and unanimously supported by the faculty. All these core values can be applied to thoracic surgical residency practices. Fostering leadership through edu- 


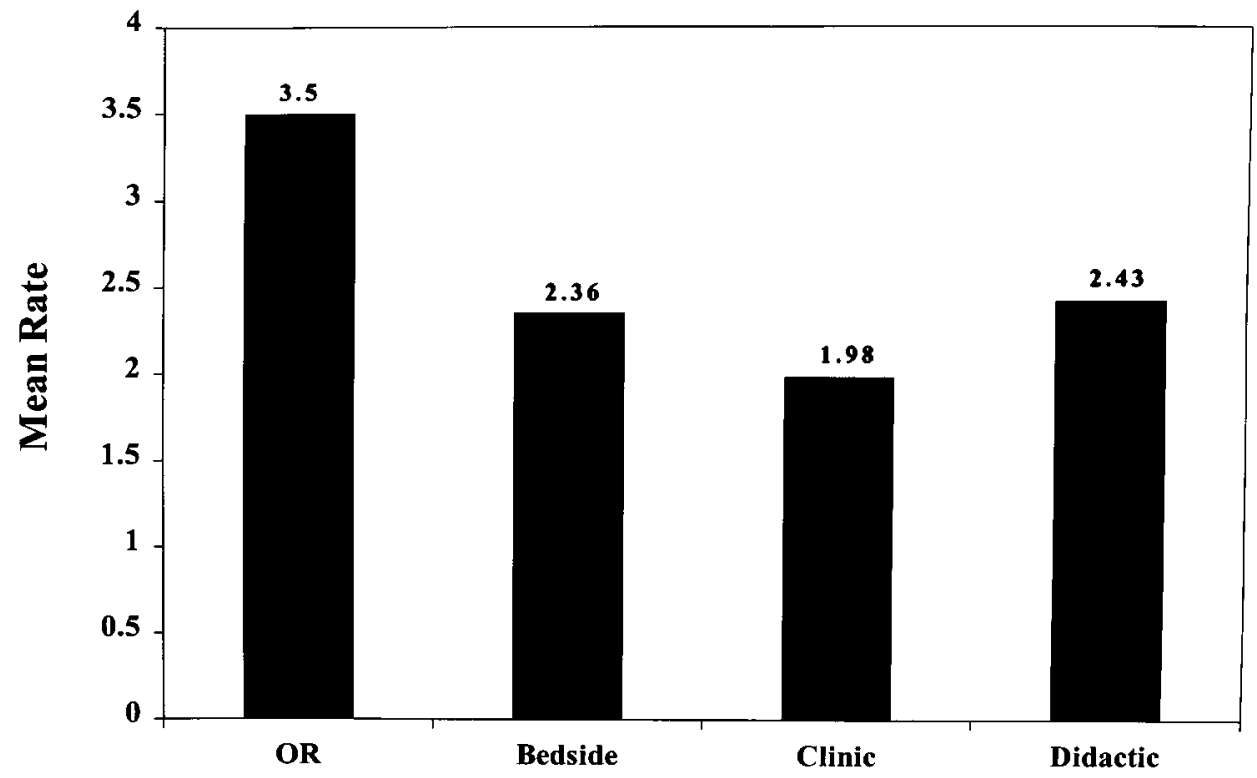

Resident Survey performed by Dr. Shemin

Fig 1. Average teaching quality of thoracic surgery residency programs.

Table I. Fundamentals of a good residency program

Place the patient first

Establish teaching and education as a priority

Respect the individual

Create a collegial atmosphere

Maintain an adequate volume

Provide evaluation cation and teaching is particularly important for the future of our specialty. The last core value is particularly pertinent to residency programs. A residency training program should develop its own core values, but 1 consistent value embedded in the fabric of all training programs is the desire and goal of fostering leadership through education and teaching.

General guidelines for progressive teaching responsibilities include careful selection of operative cases, appropriate progression of responsibility, becoming an excellent first assistant, and knowing your own limitations. There is no bigger advocate of this training philosophy then Dr Norman E. Shumway. In his 1987 AATS Presidential Address entitled, "Some Thoughts From the Other Side of the Table or the last Presidential Address," he discussed the role of the first assistant in the teaching of residents. He said in reference to the performance of an operation, "Most of you know by now that the most difficult thing about
Table II. Johns Hopkins University School of Medicine core values

Unwavering commitment to excellence

Integrity

Respect for the individual

Dedication to discovery and innovation

Foster leadership through education and teaching

surgery-even heart surgery-is getting the chance to do it." He continued his talk by saying, "Certainly it cannot matter as much who does the operation, as how it is done." Having experienced both types of training, Dr Shumway developed, nurtured and advanced the "Stanford Technique" for teaching residents. He prided himself on being 1 of the world's best first assistants.

\section{Creating the proper operating room atmosphere}

The development of a collegial environment with dedicated protocols and standardized practices is essential for training purposes and optimal patient outcomes. The cooperation of multiple individuals in a cardiac operating room is essential and mandatory to maintain excellent results.

Cooperate is defined by Webster's 9th New Collegiate Dictionary as (1) to act or work with another or others; act together; (2) to associate with another or others for 
mutual benefit. Cooperation leads to improvement through effective interaction and discussion.

In an article by Clemmer and associates, ${ }^{1}$ the authors discuss 5 scientific grounded methods to foster cooperation. These include to (1) develop a shared purpose, (2) create an open, safe environment, (3) include all those who share the common purpose and encourage diverse viewpoints, (4) learn how to negotiate agreements, and (5) insist on fairness and equity in applying rules. It is easy to see how all of these methods would pertain to a thoracic or cardiac surgery operating room.

Airline pilots are an analogous group of individuals. The airline industry has spent millions of dollars investigating and developing the proper environment in the cockpit of an airplane. This has been formalized in what is known in the airline industry as "crew or cockpit resource management," which refers to intensive training of airline crews to insure highly cooperative norms and behaviors regarding mutual assistance and exchange of information as mainstays of safety. ${ }^{2}$

The airline industry has insisted on the establishment of the proper culture in the cockpit that is conducive to unmitigated communication. Individuals are expected and encouraged to make critical comments and are praised for their actions. There is also a voluntary and confidential safety reporting system, the Aviation Safety Reporting System, through which pilots are granted immunity when they are involved in incidents in which safety is compromised. This allows the accumulation of data that leads to performance improvement in this highly technical field that is prone to human error.

It is estimated that between $70 \%$ and $80 \%$ of commercial airline accidents are due to human factors. Most of these human errors are related to communication failures. Sociologic barriers tend to inhibit the communication of crucial information against the authority gradient, for example, from a navigator or copilot to a pilot. This has direct application to our operating rooms in that an analogous scenario would be the communication of crucial information from a resident to an attending surgeon, from a perfusionist to a resident or attending, or from a nurse to an attending surgeon. ${ }^{3}$ This pertains directly to teaching surgery because it is as important to teach residents how to prevent complications as it is to teach them how to tie a knot, place a stitch, or recognize a cardiac or pulmonary abnormality.

For effective teaching and training in the operating room, it is essential to create an open and safe environment that acknowledges and encourages direct and open communication among all disciplines. In particu- lar, it is important to accept suggestions from all personnel and to eliminate the "authority gradient" in the operating room. The attending surgeon has to be the leader of cooperation in the operating room.

\section{Design of a teaching program}

A residency program has to have graded responsibility as 1 of its components. The attending physician can effectively control the operation from the left-hand side of the table. Confidence in one's myocardial protection and recognizing the graded responsibility of operative techniques are necessary components to becoming an excellent teacher in the operating room. It is important to do the operation correctly the first time with no compromise on perfection. One should expect resident learning to occur from case to case with periodic evaluations.

Although graded responsibility is subjective, one can judge the residents' technical skills when he or she is the first assistant. Appropriate operative cases can then be selected. Various parts of the operation can be introduced either individually or sequentially depending on the operative experience. These can include sternotomy, cannulation, arteriotomies, suturing, and closing. An effective teacher directs the sites of cannulation or the placement of sutures and explains each step of the procedure in terms of sequence and importance. All potential adverse scenarios and specific treatments should also be explained for completeness. Many of these scenarios should be centered around potential pitfalls in operative techniques.

Resident evaluation is an important component of a residency training program. Specific critiques should always be done in private. In addition to the critical assessment of the technical performance of the resident, positive feedback should also be provided. Resident evaluation of the faculty is also necessary to maintain a high programmatic level of education and teaching. These evaluations are occasionally difficult to solicit but are important to provide appropriate feedback to improve individual performance and the overall residency program. In response to my request to comment on what are the essentials of a good residency program, 1 resident responded, "Attending patience, patience, patience. There is no substitute." Another senior resident said, "Good relationships breed loyalty, inspire hard work, and provide role models."

Future considerations for teaching surgery will certainly involve simulators. These have been used effectively for training airline pilots for the past several years. Whenever a pilot switches aircraft, he or she must spend hours in the simulator, flying the new aircraft before an actual flight takes place. Although sur- 
gical simulators are in their early development, with the ever-increasing advancement of biomedical technology, these will become available for the education and training of surgical residents of all types. In addition to providing effective training tools, they will also be used to plan an operation and to provide useful information during the course of an operation.

As crew or cockpit resource management has pointed out, most human errors are due to system problems rather than individually incompetent people. ${ }^{4}$ This is similarly analogous to the healthcare environment. Future initiatives that apply the principles of cockpit or crew resource management will be used in the surgical specialties for the purposes of performance improvement. Error in medicine has been documented for several years. ${ }^{4}$ When the causes are investigated, it is found that most iatrogenic injuries are due to errors, many of which are preventable. The development of appropriate preventive systems will decrease the amount of error in medicine.

The National Patient Safety Foundation has a general goal of improvement of patients' safety by an extensive analysis of preventable injury within the healthcare system. ${ }^{5}$ Through its research grant program, it has made grants of $\$ 100,000$ available to sponsor medical and scientific investigators in a concerted effort to analyze causes of adverse events, errors, or failures within the healthcare system. Through scientific research and the application of the principles of crew resource man- agement, human error can be minimized, and performance improvement can be enhanced.

Teaching surgery is an art as well as a science. Although it seems redundant to state that a residency training program should have training residents as 1 of its core values, it cannot be stressed enough how important it is to make this a priority. Residents are not in the program merely to provide service. The training of residents has to be balanced with the other missions of patient care and research. Thoracic surgery residents must also be taught, in addition to technical and clinical proficiency, the management skills necessary to lead "crews" of health care providers. Without question, it provides immense personal gratification as residents develop their skills and maturity in and out of the operating room. Finally, the ultimate goal of a residency training program is to foster the development of the future leaders in our specialty.

\section{REFERENCES}

1. Clemmer TP, Spuhler VJ, Berwick DM, et al. Cooperation: the foundation of improvement. Ann Intern Med 1998;28:1004-9.

2. Weiner EL, Kanki BG, Helmreich RL, editors. Cockpit resource management. San Diego; Academic Press, 1993.

3. Helmreich RI, Schaefer HG. Team performance in the operating room. In: Bogner MS, editor. Human error in medicine. Hillside (NJ): Lawrence Erlbaum; 1994.

4. Leape LL. Error in medicine. JAMA 1994;272:1851-7.

5. Kern KA. The national patient safety foundation: what it offers surgeons. Bull Am Coll Surg 1998;83:24-7. 